\title{
BMJ Open Antipsychotic initiation among adults with intellectual and developmental disabilities in Ontario: a population- based cohort study
}

\author{
Tara Gomes, ${ }^{1,2,3,4}$ Wayne Khuu, ${ }^{2}$ Mina Tadrous, ${ }^{1,2,3}$ Simone Vigod, ${ }^{2,4,5,6}$ \\ Virginie Cobigo, ${ }^{2,7}$ Yona Lunsky ${ }^{2,5,8}$
}

To cite: Gomes T, Khuu W, Tadrous M, et al. Antipsychotic initiation among adults with intellectual and developmental disabilities in Ontario: a population-based cohort study. BMJ Open 2019;9:e028125. doi:10.1136/ bmjopen-2018-028125

- Prepublication history for this paper and additional material is published online only. To view please visit the journal online (http://dx.doi.org/10.1136/ bmjopen-2018-028125)

Received 23 November 2018 Revised 29 May 2019 Accepted 7 June 2019
Check for updates

\section{(C) Author(s) (or their} employer(s)) 2019. Re-use permitted under CC BY-NC. No commercial re-use. See rights and permissions. Published by BMJ.

For numbered affiliations see end of article.

Correspondence to

Tara Gomes; gomest@smh.ca

\section{ABSTRACT}

Objectives To describe factors associated with initiating antipsychotics and patterns of persistence to antipsychotic therapy in a large cohort of adults with intellectual and developmental disabilities.

Design Population-based cohort study.

Setting Ontario, Canada.

Participants Adults with intellectual and developmental disabilities (IDD) in Ontario.

Outcome measures We used multivariable logistic regression to investigate patient characteristics associated with antipsychotic initiation. Patient characteristics studied included sociodemographic characteristics, measures of clinical comorbidity and health service use.

Results Among 39244 individuals eligible for this study, $6924(17.6 \%)$ initiated an antipsychotic over the accrual window, of whom 1863 (26.9\%) had no psychiatric diagnosis in the prior 2 years. A number of factors were significantly associated with antipsychotic initiation, including male gender, residence in a group home, prior use of benzodiazepines, antidepressants or cognitive enhancers, a recent emergency department visit or mental health hospitalisation and a visit to a psychiatrist or family physician in the prior 90 days. In a secondary analysis, the association between antipsychotic initiation and age, prior diagnosis of diabetes or myocardial infarction and polypharmacy differed slightly on the basis of whether an individual had a previously diagnosed psychiatric disorder. Conclusions Factors associated with the initiation of an antipsychotic differ according to the presence of a psychiatric diagnosis. Given the long duration of antipsychotic use in this population, future research is needed to understand the appropriateness of antipsychotic initiation among adults with IDD and the safety implications of long-term use of these products.

\section{INTRODUCTION}

There is a growing recognition that use of antipsychotics is high among adults with intellectual and developmental disabilities (IDD) internationally, with rates ranging between $21 \%$ and $45 \% .^{1-7}$ In Canada, a recent study found that $39 \%$ of adults with IDD are treated with these medications. ${ }^{8}$ For some, antipsychotics are

\section{Strengths and limitations of this study}

- This study uses linked population-based data allowing us to study medication use and health services utilisation among a large cohort of adults with intellectual and developmental disabilities (IDD), regardless of how they interact with the health system.

- We relied on diagnostic codes from administrative claims databases in the prior 2 years to define the presence of psychiatric diagnoses. Although this definition is used regularly in Ontario, it has not been validated in our population of adults with IDD, and therefore it is possible that some misclassification occurred.

- We cannot confirm whether antipsychotics that were dispensed to individuals without a psychiatric diagnosis were being prescribed to manage behavioural concerns, and therefore, cannot draw definitive conclusions about the use of this class of medications for this indication in our study.

- We determined residence in a group home setting using data from 2009 and assumed that this did not change over the study period. Although residence in these settings is relatively stable in Ontario, it is possible that living arrangements for some members of our cohort could have changed over the study period.

- We are unable to study some variables that may be associated with antipsychotic initiation, including severity of intellectual disability, history of challenging behaviour and family and paid caregiver level of stress and attitudes towards medication use.

prescribed to treat psychiatric disorders, but in many cases, they are used as a means to manage behaviour in the absence of a comorbid mental health disorder, ${ }^{3}$ despite limited evidence of their efficacy for this purpose. ${ }^{3} 9$ Long-term use of antipsychotics has been associated with adverse effects including sedation, extrapyramidal side effects, anticholinergic effects, cardiometabolic problems including diabetes, hypertension, thromboembolism, stroke and, rarely, sudden death. ${ }^{31011}$ Given these risks, 
the high rate of antipsychotic use among adults with IDD is concerning, and even more so because this population has high rates of comorbid health issues, and may have difficulty reporting side effects, thus delaying medical intervention. 1213

Despite many studies documenting high rates of antipsychotic use in this population, few have focused specifically on patient factors associated with initiation of these medications. Given the frequent use of antipsychotics in this population, concerns around their safety and off-label use for behavioural management, more evidence is needed on patient characteristics associated with the initiation and duration of use of these medications among adults with IDD. The objective of this study was to describe factors associated with initiating antipsychotics and patterns of persistence to antipsychotic therapy in a large cohort of adults with IDD.

\section{MATERIAL AND METHODS}

We conducted a population-based cohort study that followed a cohort of 66484 Ontario adults with IDD aged 18-64 years as of 1 April 2009 who received disability support benefits. All residents of Ontario have universal, government-funded access to physician and hospital services. Furthermore, this population has access to publicly funded medications through the disability support programme. IDD was defined to be consistent with Ontario legislation to include terms such as mental retardation/intellectual disability, autism and other pervasive developmental disorders, fetal alcohol syndrome and various chromosomal disorders known to be aetiological factors of IDD. This cohort was generated by linking data from provincial health and social services data in Ontario, Canada, and defined IDD on the basis of: (1) the presence of IDD diagnostic codes in two or more physician claims since database inception (1991), (2) the presence of IDD diagnostic codes on one or more hospital claims since database inception (1988) or (3) IDD recorded as a diagnosis leading to eligibility for disability benefits from social services. ${ }^{13}$ This project was completed as part of the Health Care Access Research and Developmental Disabilities Program and the Ontario Drug Policy Research Network.

\section{Data sources}

We used the Registered Persons Database to determine patient demographics and vital statistics and identified all prescription drugs dispensed from community pharmacies using the Ontario Drug Benefit claims database. We used the Canadian Institute for Health Information's Discharge Abstract Database, Same Day Surgery database, National Ambulatory Care Reporting System and Ontario Mental Health Reporting System to identify diagnoses and procedures from inpatient hospitalisations, same-day surgeries, emergency department visits and mental health hospitalisations, respectively. We used the Ontario Health Insurance Program claims database to determine outpatient health services utilisation, including primary care and specialist visits and related billings. Finally, we used three validated databases, the Ontario Diabetes Database, ${ }^{14}$ the Ontario Hypertension Database ${ }^{15}$ and the Ontario Myocardial Infarction Database ${ }^{16}$ to determine past diagnoses of diabetes, hypertension and myocardial infarction, respectively. These datasets were linked using unique, encoded identifiers, were analysed at ICES (www.ices.on.ca) and are used regularly to assess the drug utilisation, safety and policy in the Ontario healthcare system. ${ }^{8}{ }^{8-19}$ More details on these data sources can be found in online supplementary appendix etable 1. ICES is a prescribed entity under section 45 of Ontario's Personal Health Information Protection Act (PHIPA), which enables analysis and compilation of statistical information related to the management, evaluation and monitoring of, allocation of resources to, and planning for the health system. Section 45 authorises health information custodians to disclose personal health information to a prescribed entity, like ICES, without consent for such purposes. Therefore, this study did not require informed consent.

\section{Outcome measure}

From within the cohort of adults with IDD, we identified our primary outcome as individuals who were newly prescribed an antipsychotic (online supplementary appendix etable 2) between 1 April 2010 and 31 March 2016. We defined antipsychotic initiation as any antipsychotic prescription filled during the study period with no antipsychotic prescribed in the prior year. Individuals not prescribed an antipsychotic over our study period or in the year prior were considered unexposed. The first antipsychotic prescription date was the index date for the exposed cohort. For the unexposed, we randomly assigned an index date following the same distribution as of dates observed among antipsychotic users.

\section{Patient characteristics}

We explored sociodemographic characteristics, measures of clinical comorbidity and health service use as potential factors associated with antipsychotic initiation in this population. Sociodemographic characteristics included age, sex, income quintile, rural location of residence and whether the individual resided in a group home. We defined prior psychiatric diagnoses using methods published previously. ${ }^{20}{ }^{21}$ Specifically, we defined a psychiatric diagnosis as either a major mental disorder (schizophrenia, non-schizophrenia psychotic disorder, bipolar disorder or major depressive disorder) or other mental disorder (other depressive disorders, anxiety, somatoform, dissociative, psychosomatic, personality disorders, adjustment disorders and disorders of conduct or impulsivity) diagnosed in the 2 years up to and including the index date using inpatient and outpatient datasets (see online supplementary appendix etables 3 and 4). ${ }^{20}{ }^{21}$ We used the Charlson Comorbidity Index as a measure of overall medical comorbidity using hospital claims in the prior 3 years $^{22}$ and reported the presence of any prior diagnosis of diabetes, hypertension or myocardial infarction using validated databases described above. Measures 
of medication use in the prior year included total number of drugs dispensed, and any exposure to antidepressants, benzodiazepines, lithium or antiepileptic drugs, stimulants or cognitive enhancers. We included several measures of health services utilisation, including inpatient hospitalisations (medical and psychiatric) and emergency department visits in the prior 2 years, number of physician visits in the prior 1 year and recent visits to a specialist (psychiatrist or neurologist; prior 90 days).

\section{Duration of antipsychotic use}

Among new antipsychotic users, we characterised prescriber specialty for the initial antipsychotic prescription (psychiatrist, family physician, other specialist and unknown) and the initial antipsychotic dose (in chlorpromazine equivalents). In cases where individuals were initiated on multiple antipsychotics, the total dose was calculated as the sum of the doses on all prescriptions dispensed at initiation. We also characterised a period of ongoing use of antipsychotics among new users of these drugs to investigate patterns of drug discontinuation over a 1-year follow-up period. We defined ongoing antipsychotic use on the basis of a prescription refill within 180 days of a previous prescription. If no such refill was found, the individual was flagged as having discontinued antipsychotic therapy, and their discontinuation date was set as the date on which the last prescription filled would have been completed (ie, date of dispensing plus days supply). In this analysis, we followed people forward until the first of drug discontinuation or a censoring event (death, end of observation period (31 March 2016) or 1 year of maximum follow-up).

\section{Statistical analysis}

In the primary analysis, we compared baseline characteristics of exposed and unexposed individuals using standardised differences, which are used in large population-based studies where the size of the population can influence $p$ values. A standardised difference above 0.10 is considered meaningful. ${ }^{23}$ We used multivariable logistic regression to investigate patient characteristics associated with antipsychotic initiation. We included all baseline covariates described above in the model. All continuous variables were first modelled as such. If a non-linear relationship between these variables and the log-odds of the outcome was suspected, then we categorised these variables and modelled the categorical variables instead. Regression models were created for the overall cohort and stratified into individuals with and without a psychiatric diagnosis at baseline. Missing values were categorised separately in the models.

For antipsychotic users only, we generated KaplanMeier estimates for time to drug discontinuation and reported the median time to discontinuation and the proportion of the population who remained on therapy for at least 6 and 12 months. All analyses were conducted overall and stratified by psychiatric diagnosis at baseline. We used the log-rank test to measure differences in time to discontinuation and $\chi^{2}$ tests to compare initial antipsychotic prescription characteristics between those with and without a psychiatric diagnosis at baseline. We defined statistically significant results as those with a type 1 error rate less than $5 \%$.

\section{Patient and public involvement}

Patients and the public were not involved in the design or conduct of this study.

\section{RESULTS}

Among the 66484 adults with IDD in Ontario, 20316 were prescribed an antipsychotic over the accrual period, among whom 6924 met eligibility criteria and were new antipsychotic users. The remaining 46168 did not have a record of an antipsychotic prescription through the public drug programme over the accrual period, among whom 32320 met our eligibility criteria (see online supplementary appendix efigure 1 for full details of exclusions). In general, adults with IDD initiating antipsychotics were similar to the unexposed group with respect to mean age ( 41.0 vs 40.7 years), sex (56.1\% vs $56.7 \%$ male), neighbourhood income $(32.7 \%$ in lowest income quintile in both groups), rurality $(84.2 \%$ vs $82.0 \%$ residing in urban areas) and residence in a group home setting $(11.1 \%$ vs $9.1 \%$; table 1). There were several important differences between these groups in prior medication use, prior health services utilisation and clinical comorbidities. For example, adults initiating antipsychotics were more likely to have recently been prescribed antidepressants $(43.2 \%$ vs $15.1 \%$ ) and benzodiazepines (28.8\% vs $11.4 \%$ ), were more likely to have visited an emergency department in the past 2 years $(68.8 \%$ vs $42.6 \%)$ and had a higher prevalence of major mental illness (31.3\% vs $2.6 \%$ ) compared with the unexposed group (table 1).

In the multivariable model, all continuous variables were modelled as categories, with the exception of the number of visits to a physician in the prior year (c-statistic for model, 0.862). Important factors associated with antipsychotic initiation were being male (adjusted OR (aOR) $1.21,95 \%$ CI 1.13 to 1.29 ), residence in a group home (aOR $1.20,95 \%$ CI 1.08 to 1.34 ), past use of antidepressants (aOR 1.96, 95\% CI 1.82 to 2.12 ), benzodiazepines (aOR $1.60,95 \% \mathrm{CI} 1.46$ to 1.74 ) and cognitive enhancers (aOR 5.10, 95\% CI 3.78 to 6.87 ), an ED visit (aOR $1.43,95 \%$ CI 1.33 to 1.54 ) or mental health hospitalisation (aOR 1.84, 95\% CI 1.61 to 2.09) in the past 2 years and a visit to a psychiatrist (aOR 4.82, 95\% CI 4.31 to 5.40 ) or family physician (aOR $1.67,95 \%$ CI 1.55 to 1.79 ) in the previous 90 days (table 2). As expected, both prior diagnosis of a major mental disorder (aOR 5.18, $95 \% \mathrm{CI} 4.65$ to 5.77) and other mental disorder (aOR $3.18,95 \%$ CI 2.96 to 3.41 ) were strongly associated with antipsychotic use. Several factors were associated with a lower odds of antipsychotic initiation in the multivariable model, including being aged 30 to 45 (aOR $0.84,95 \%$ CI 0.77 to 0.92 compared with those aged $18-29$ years), past 
Table 1 Characteristics of adults with intellectual and developmental disabilities, stratified on basis of antipsychotic initiation (1 April 2010-31 March 2016)

\begin{tabular}{|c|c|c|c|}
\hline & $\begin{array}{l}\text { Initiated antipsychotic } \\
\mathrm{n}=6924\end{array}$ & $\begin{array}{l}\text { No antipsychotic use } \\
n=32320\end{array}$ & $\begin{array}{l}\text { Standardised } \\
\text { difference* }^{*}\end{array}$ \\
\hline \multicolumn{4}{|l|}{ Demographic characteristics } \\
\hline Age, mean (SD) & $41.0(13.9)$ & $40.7(13.2)$ & 0.02 \\
\hline 18-29, n (\%) & $1986(28.7)$ & $8805(27.2)$ & 0.03 \\
\hline 30-45, n (\%) & $2135(30.8)$ & $10885(33.7)$ & 0.06 \\
\hline $46+, \mathrm{n}(\%)$ & $2803(40.5)$ & $12630(39.1)$ & 0.03 \\
\hline Male, n (\%) & $3886(56.1)$ & $18329(56.7)$ & 0.01 \\
\hline Urban residence, n (\%) & $5830(84.2)$ & $26512(82.0)$ & 0.06 \\
\hline \multicolumn{4}{|l|}{ Income quintile, n (\%) } \\
\hline 1 & $2267(32.7)$ & $10572(32.7)$ & 0.00 \\
\hline 2 & $1476(21.3)$ & $6958(21.5)$ & 0.01 \\
\hline 3 & $1175(17.0)$ & $5539(17.1)$ & 0.00 \\
\hline 4 & 1065 (15.4) & $4803(14.9)$ & 0.01 \\
\hline 5 & $893(12.9)$ & $4031(12.5)$ & 0.01 \\
\hline Missing & $48(0.7)$ & 417 (1.3) & 0.06 \\
\hline Residing in group home & 768 (11.1) & $2933(9.1)$ & 0.07 \\
\hline \multicolumn{4}{|l|}{ Medication use } \\
\hline $\begin{array}{l}\text { Number of drugs dispensed in past year, median } \\
\text { (IQR) }\end{array}$ & $5(1-9)$ & $2(0-6)$ & 0.40 \\
\hline \multicolumn{4}{|l|}{ Past use of other medications (prior 1 year), $\mathrm{n}(\%)$} \\
\hline Antidepressants & $2994(43.2)$ & $4876(15.1)$ & 0.65 \\
\hline Benzodiazepines & $1997(28.8)$ & $3692(11.4)$ & 0.45 \\
\hline Lithium or antiepileptic drug & $622(9.0)$ & $2647(8.2)$ & 0.03 \\
\hline Stimulant & $215(3.1)$ & $372(1.2)$ & 0.14 \\
\hline Cognitive enhancer & $134(1.9)$ & $108(0.3)$ & 0.15 \\
\hline \multicolumn{4}{|l|}{ Health services utilisation } \\
\hline Hospitalised in past 2 years, $n(\%)$ & $1645(23.8)$ & $3338(10.3)$ & 0.36 \\
\hline ED visit in past 2 years, $\mathrm{n}(\%)$ & $4767(68.8)$ & $13776(42.6)$ & 0.55 \\
\hline Mental health hospitalisation in past 2 years, $\mathrm{n}(\%)$ & $1669(24.1)$ & $819(2.5)$ & 0.67 \\
\hline $\begin{array}{l}\text { Number of physician visits in past year, median } \\
\text { (IQR) }\end{array}$ & $6(3-12)$ & $3(1-6)$ & 0.66 \\
\hline \multicolumn{4}{|l|}{ Specialist visits in past 90 days, $n(\%)$} \\
\hline Psychiatrists & $2115(30.5)$ & $648(2.0)$ & 0.84 \\
\hline Family practice & $4979(71.9)$ & $15089(46.7)$ & 0.53 \\
\hline Neurologist & $343(5.0)$ & $820(2.5)$ & 0.13 \\
\hline \multicolumn{4}{|l|}{ Comorbidities, n (\%) } \\
\hline \multicolumn{4}{|l|}{ Charlson score (using 3 years of hospitalisation data) } \\
\hline No hospitalisation & $4963(71.7)$ & $27887(86.3)$ & 0.36 \\
\hline 0 & $1172(16.9)$ & $2939(9.1)$ & 0.23 \\
\hline 1 & $296(4.3)$ & $759(2.3)$ & 0.11 \\
\hline $2+$ & $493(7.1)$ & $735(2.3)$ & 0.23 \\
\hline \multicolumn{4}{|l|}{ Psychiatric diagnosis in the past 2 years, $n(\%)$} \\
\hline Major mental illness & 2169 (31.3) & $846(2.6)$ & 0.83 \\
\hline Other psychiatric diagnosis & $4654(67.2)$ & $6437(19.9)$ & 1.09 \\
\hline Both of the above & $1762(25.4)$ & $494(1.5)$ & 0.75 \\
\hline
\end{tabular}




\begin{tabular}{|c|c|c|c|}
\hline & $\begin{array}{l}\text { Initiated antipsychotic } \\
n=6924\end{array}$ & $\begin{array}{l}\text { No antipsychotic use } \\
n=32320\end{array}$ & $\begin{array}{l}\text { Standardised } \\
\text { difference* }^{*}\end{array}$ \\
\hline Neither of the above & 1863 (26.9) & $25531(79.0)$ & 1.22 \\
\hline Prior diagnosis of hypertension & $1358(19.6)$ & 5697 (17.6) & 0.05 \\
\hline Prior myocardial infarction & $56(0.8)$ & $261(0.8)$ & 0.00 \\
\hline
\end{tabular}

*All comparisons with standardised difference $>0.10$, indicating a meaningful difference between groups, are presented in bold font.

use of lithium or antiepileptic drugs (aOR 0.84, 95\% CI 0.74 to 0.94 ) and each of diagnoses of diabetes mellitus (aOR $0.85,95 \%$ CI 0.76 to 0.94 ), hypertension (aOR 0.76 , $95 \%$ CI 0.69 to 0.83 ) or a history of myocardial infarction (aOR $0.54,95 \%$ CI 0.37 to 0.79 ).

Among the 6924 individuals who initiated an antipsychotic, $1863(26.9 \%)$ had no evidence of a psychiatric diagnosis in the 2 years preceding the index prescription. In analyses stratified by presence or absence of a psychiatric diagnosis in the 2 years preceding the index date, the multivariable models were similar (c-statistics, 0.753 and 0.762 , respectively). Among those with a psychiatric diagnosis, older individuals (aged 46 and older) were less likely than those aged 18-29 to start an antipsychotic (aOR $0.87,95 \%$ CI 0.77 to 0.97 ). The opposite pattern was observed for those without a psychiatric diagnosis, whereby older adults were more likely to start antipsychotics (aOR 1.19, 95\% CI 1.03 to 1.38). Furthermore, those without a psychiatric diagnosis were less likely to start antipsychotics if they had a history of diabetes or myocardial infarction; however, this was not the case among those with a psychiatric diagnosis. Finally, there were important differences in the relationship with polypharmacy measures between groups. Among individuals with no psychiatric diagnosis, those taking two or more medications in the previous year were more likely to initiate antipsychotics compared with those with no medication use in the prior year (range of aOR 1.23-1.24). In contrast, among those with a psychiatric diagnosis, higher degrees of polypharmacy were protective against initiating an antipsychotic (range of aOR 0.62-0.78; table 3).

We also observed differences in initial antipsychotic dose and prescriber specialty between those with and without a psychiatric diagnosis (table 3). In general, those without a psychiatric diagnosis were more likely to receive their first antipsychotic prescription from a family physician $(65.3 \%$ vs $47.4 \%$; $\mathrm{p}<0.001)$ and less likely to receive their first prescription from a psychiatrist $(9.6 \%$ vs $36.3 \%$; $\mathrm{p}<0.001)$. Initial antipsychotic dose was similar between patient groups (median $80 \mathrm{mg}$ chlorpromazine equivalents; IQR 40-200 mg), but those with a psychiatric diagnosis escalated to a higher maximum antipsychotic dose compared with those with no such diagnosis $(150 \mathrm{mg}$ vs $110 \mathrm{mg}$ chlorpromazine equivalents; $\mathrm{p}<0.001$ ).

Overall, the median time to antipsychotic discontinuation among adults with IDD was 354 days; however, this also differed significantly according to prior psychiatric diagnosis $(\mathrm{p}<0.001)$. Specifically, those with a prior psychiatric diagnosis were more likely to continue therapy for 6 months $(60.8 \%$ vs $50.7 \%, \mathrm{p}<0.001)$ and 12 months $(50.7 \%$ vs $41.8 \%, \mathrm{p}<0.001)$ compared with those with no such diagnosis (table 3 ).

\section{DISCUSSION}

In this population-based study of nearly 40000 previously untreated adults with IDD residing in Ontario, Canada, we found that approximately 1 in 6 initiated an antipsychotic medication over our 6-year study period. Furthermore, over one-quarter of those starting an antipsychotic had no indication of a psychiatric condition being diagnosed in the prior 2 years and had a lower maximum dose achieved. This suggests that antipsychotics may be used frequently in adults with IDD for off-label indications, such as managing behavioural issues, sedation or sleep. In general, independent factors associated with antipsychotic initiation included being male, residing in a group home, prior use of psychiatric medications, high health services utilisation and previous psychiatric diagnoses, while having diabetes, hypertension or a previous myocardial infarction reduced the chance that an antipsychotic would be initiated. Although there was little difference in variables associated with antipsychotic initiation when considering those with and without psychiatric diagnoses separately, one important finding is that people without a psychiatric diagnosis who had received multiple other medications in the year prior were more likely to be started on an antipsychotic, whereas such polypharmacy was protective against antipsychotic initiation among people with psychiatric diagnoses. Although the specific drivers of this finding are unknown, future research should investigate the differential role of polypharmacy in antipsychotic initiation among those with and without a concurrent psychiatric diagnosis.

The patterns observed in this study are generally consistent with previously published research exploring antipsychotic use in the IDD population. For example, a study by Sheehan $e t a l^{7}$ similarly found that major mental illness was associated with increased likelihood of initiating antipsychotics among adults with IDD, although they also identified a subgroup of the population who initiated antipsychotics in the absence of a psychiatric diagnosis. 
Table 2 Factors associated with initiation of antipsychotics among adults with intellectual and developmental disabilities, overall and stratified by presence of mental health diagnosis at time of initiation

\section{No psychiatric \\ Overall diagnosis \\ Adjusted ORs (95\% Cl) Adjusted ORs (95\% Cl) \\ Psychiatric diagnosis \\ Adjusted ORs $(95 \% \mathrm{Cl})$}

Demographic characteristics

Age category (years)

\begin{tabular}{|llll}
\hline $18-29$ & 1.00 & 1.00 & 1.00 \\
\hline $30-45$ & $0.84(0.77$ to 0.92$)$ & $0.88(0.76$ to 1.02$)$ & $0.86(0.78$ to 0.96$)$ \\
\hline $46+$ & $0.96(0.88$ to 1.05$)$ & $1.19(1.03$ to 1.38$)$ & $0.87(0.77$ to 0.97$)$ \\
\hline $\begin{array}{l}\text { Male } \\
\text { Urban residence }\end{array}$ & $1.21(1.13$ to 1.29$)$ & $1.27(1.14$ to 1.41$)$ & $1.15(1.06$ to 1.25$)$ \\
\hline $\begin{array}{l}\text { Residing in group home } \\
\text { Neighbourhood income quintile }\end{array}$ & $0.97(0.89$ to 1.05$)$ & $0.89(0.78$ to 1.01$)$ & $1.06(0.94$ to 1.19$)$ \\
\hline 1 & $1.20(1.08$ to 1.34$)$ & $1.11(0.95$ to 1.29$)$ & $1.12(0.96$ to 1.29$)$ \\
\hline 2 & 1.00 & 1.00 & 1.00 \\
\hline 3 & $1.05(0.96$ to 1.15$)$ & $0.99(0.86$ to 1.15$)$ & $1.10(0.98$ to 1.23$)$ \\
\hline 4 & $1.13(1.02$ to 1.24$)$ & $1.10(0.95$ to 1.28$)$ & $1.14(1.01$ to 1.29$)$ \\
\hline 5 & $1.16(1.04$ to 1.28$)$ & $1.20(1.03$ to 1.40$)$ & $1.14(1.00$ to 1.30$)$ \\
\hline Missing & $1.19(1.07$ to 1.32$)$ & $1.25(1.06$ to 1.46$)$ & $1.09(0.95$ to 1.25$)$ \\
\hline
\end{tabular}

Medication use

Number of drugs dispensed in past year

\begin{tabular}{|c|c|c|c|}
\hline 0 & 1.00 & 1.00 & 1.00 \\
\hline 1 & 0.98 (0.86 to 1.12$)$ & 1.09 (0.88 to 1.35$)$ & 0.78 (0.65 to 0.92$)$ \\
\hline $2-5$ & 0.97 (0.88 to 1.08$)$ & 1.23 (1.04 to 1.45$)$ & 0.66 (0.58 to 0.76$)$ \\
\hline $5+$ & 0.95 (0.84 to 1.08$)$ & 1.24 (1.02 to 1.51$)$ & 0.62 (0.53 to 0.73$)$ \\
\hline \multicolumn{4}{|l|}{ Past use of other medications (prior 1 year) } \\
\hline Antidepressants & 1.96 (1.82 to 2.12$)$ & 2.80 (2.47 to 3.18$)$ & 1.57 (1.43 to 1.73$)$ \\
\hline Benzodiazepines & $1.60(1.46$ to 1.74$)$ & 2.08 (1.82 to 2.39$)$ & 1.46 (1.32 to 1.62$)$ \\
\hline Lithium or antiepileptic drugs & 0.84 (0.74 to 0.94$)$ & 0.79 (0.67 to 0.94$)$ & 0.83 (0.70 to 0.98$)$ \\
\hline Stimulants & $1.20(0.96$ to 1.49$)$ & 2.19 (1.45 to 3.29$)$ & 0.96 (0.75 to 1.22$)$ \\
\hline Cognitive enhancers & 5.10 (3.78 to 6.87$)$ & 5.49 (3.80 to 7.94$)$ & 3.56 (2.21 to 5.72$)$ \\
\hline \multicolumn{4}{|l|}{ Health services utilisation } \\
\hline Hospitalisation in prior 2 years & 1.14 (0.96 to 1.36$)$ & $1.31(1.00$ to 1.71$)$ & 0.88 (0.70 to 1.10$)$ \\
\hline Emergency department visit in prior 2 years & $1.43(1.33$ to 1.54$)$ & 1.23 (1.09 to 1.37$)$ & 1.55 (1.41 to 1.70$)$ \\
\hline Mental health hospitalisation prior 2 years & 1.84 (1.61 to 2.09$)$ & 1.71 (1.33 to 2.21$)$ & 3.27 (2.85 to 3.76$)$ \\
\hline Number of physician visits in prior year & 1.01 (1.00 to 1.01$)$ & 1.03 (1.02 to 1.03$)$ & 1.00 (1.00 to 1.01$)$ \\
\hline \multicolumn{4}{|l|}{ Specialist visit in prior 90 days } \\
\hline Psychiatrist & $4.82(4.31$ to 5.40$)$ & 10.86 (7.87 to 15.00$)$ & 5.11 (4.56 to 5.72$)$ \\
\hline Family practice & $1.67(1.55$ to 1.79$)$ & $1.62(1.44$ to 1.82$)$ & 1.56 (1.42 to 1.71$)$ \\
\hline Neurologist & 1.17 (0.99 to 1.39$)$ & $1.22(0.95$ to 1.56$)$ & 1.12 (0.90 to 1.40$)$ \\
\hline \multicolumn{4}{|l|}{ Comorbidities } \\
\hline \multicolumn{4}{|c|}{ Charlson score (past 3 years of hospitalisation data) } \\
\hline No hospitalisation & 1.00 & 1.00 & 1.00 \\
\hline 0 & 1.07 (0.91 to 1.27$)$ & 1.29 (1.00 to 1.66$)$ & $1.02(0.83$ to 1.25$)$ \\
\hline 1 & 1.05 (0.83 to 1.32$)$ & $0.92(0.64$ to 1.32$)$ & 1.04 (0.78 to 1.38$)$ \\
\hline $2+$ & 2.75 (2.22 to 3.42$)$ & 3.50 (2.57 to 4.75$)$ & 1.60 (1.19 to 2.15$)$ \\
\hline Major mental illness & 5.18 (4.65 to 5.77$)$ & - & - \\
\hline
\end{tabular}

Continued 


\begin{tabular}{|c|c|c|c|}
\hline & $\begin{array}{l}\text { Overall } \\
\text { Adjusted ORs }(95 \% \mathrm{Cl})\end{array}$ & $\begin{array}{l}\text { No psychiatric } \\
\text { diagnosis } \\
\text { Adjusted ORs }(95 \% \mathrm{Cl})\end{array}$ & $\begin{array}{l}\text { Psychiatric diagnosis } \\
\text { Adjusted ORs (95\% Cl) }\end{array}$ \\
\hline Diabetes & 0.85 (0.76 to 0.94$)$ & 0.83 (0.71 to 0.98$)$ & 0.91 (0.80 to 1.04$)$ \\
\hline Hypertension & 0.76 (0.69 to 0.83$)$ & 0.66 (0.57 to 0.76$)$ & $0.86(0.77$ to 0.97$)$ \\
\hline
\end{tabular}

Furthermore, several studies have highlighted psychotropic use generally ${ }^{24}$ and antipsychotics specifically ${ }^{4525}$ being more commonly prescribed to those in residential care settings compared with those living with family or independently. This may speak to increased patient complexity (eg, higher rates of psychiatric disorders or challenging behaviour) leading to residential care but may also be associated with greater stressors in those environments or prescribing attitudes of formal versus family caregivers. ${ }^{6}$ Prior studies have suggested that older adults are more likely to be prescribed antipsychotics than younger adults, ${ }^{12726}$ but this was influenced by the way in which age is grouped in each study. Our study suggests that this relationship is impacted by concurrent psychiatric diagnosis. In particular, adults over age 45 years were more likely to be prescribed antipsychotics in the absence of a psychiatric diagnosis but less likely to be prescribed these medications when there was a psychiatric diagnosis. This may be influenced by the timing at which psychiatric illnesses are commonly diagnosed, with this diagnosis typically occurring in younger adults who will therefore be initiated on antipsychotics at a younger age. It could also be suggestive of antipsychotics being used off-label for sleep disturbances in older populations as has been reported in studies of older adults with IDD. ${ }^{27}$

Our findings have important implications for clinical practice in this vulnerable population. First, we found that the likelihood of antipsychotic initiation is lower among individuals who have a prior diagnosis for a metabolic or cardiovascular condition (eg, diabetes, hypertension and myocardial infarction), which suggests that clinicians are aware of the literature regarding the potential risk factors for adverse events when prescribing this class of medications in this population ${ }^{28} 29$ and demonstrate more caution when diabetes or hypertension are already present. However, our findings also suggest that individuals taking a large number of other medications and those with a high degree of comorbidity are more likely to initiate antipsychotics and that nearly half of this population will continue their medication for at least 1 year. This is concerning given the risks associated with long-term use of antipsychotics, particularly among individuals who

Table 3 Patterns of use among adults with developmental disabilities who initiate antipsychotics between 1 April 2010 and 31 March 2016

\begin{tabular}{|c|c|c|c|c|}
\hline Characteristic & $\begin{array}{l}\text { Overall } \\
n=6924\end{array}$ & $\begin{array}{l}\text { No psychiatric } \\
\text { diagnosis } \\
n=1863\end{array}$ & $\begin{array}{l}\text { Psychiatric } \\
\text { diagnosis } \\
n=5061\end{array}$ & $P$ value \\
\hline \multicolumn{5}{|c|}{ Prescriber specialty of first antipsychotic prescription, n (\%) } \\
\hline Psychiatrist & $2015(29.1)$ & $178(9.6)$ & $1837(36.3)$ & $<0.001$ \\
\hline Family practice & $3613(52.2)$ & $1216(65.3)$ & $2397(47.4)$ & $<0.001$ \\
\hline \multicolumn{5}{|l|}{ Antipsychotic dose* } \\
\hline Dose at initiation (median, IQR) & $80(40-200)$ & $80(40-200)$ & $80(40-200)$ & 0.195 \\
\hline Maximum dose received (median, IQR) & $140(50-300)$ & $110(50-274)$ & $150(57-300)$ & $<0.001$ \\
\hline \multicolumn{5}{|c|}{ Patterns of discontinuation of any antipsychotic } \\
\hline 1 year & 3347 (48.3) & $779(41.8)$ & $2568(50.7)$ & $<0.001$ \\
\hline
\end{tabular}

* Reported as chlorpromazine equivalents.

†Cannot be estimated because more than $50 \%$ of individuals continued treatment at end of study period.

$\ddagger \mathrm{P}$ value from log-rank test. All other $\mathrm{p}$ values reported are the result of $\chi^{2}$ tests. 
may have challenges communicating with providers when experiencing adverse events. Finally, among individuals with no psychiatric diagnosis, nearly two-thirds of antipsychotics were initiated by a family physician, which is much higher than rates observed among those with psychiatric diagnoses in our study (47\%). Although this could be due to different patterns of access to specialists between these populations, this finding is consistent with those discussed in research by Holden et al, which also found that family physicians were less likely to provide psychosocial interventions and monitor medication side effects in this population compared with psychiatrists. ${ }^{1}$ Therefore, the findings of our study suggest that future research is needed to better understand indications for antipsychotic initiation and rigour of long-term medication monitoring by family physicians in this vulnerable population.

\section{Strengths and limitations}

A main strength of this study is its use of linked population-based data allowing us to study medication use and health services utilisation among a large cohort of adults with IDD, regardless of how they interact with the health system. However, there are several limitations that merit emphasis. First, we relied on diagnostic codes from administrative claims databases in the prior 2 years to define the presence of psychiatric diagnoses. Although this definition is used regularly in Ontario, it has not been validated in our population of adults with IDD, and therefore, it is possible that some misclassification occurred. However, given the differences observed in our stratified models, the findings from this study suggest that our definition of psychiatric diagnoses successfully identified two unique subpopulations in our cohort. Second, we cannot confirm whether antipsychotics that were dispensed to individuals without a psychiatric diagnosis were being prescribed to manage behavioural concerns, and therefore, we cannot draw definitive conclusions about the use of this class of medications for this indication in our study. However, the fact that individuals without a psychiatric diagnosis were also more likely to have a lower maximum dose achieved suggests that this may be the case. Furthermore, it is possible that antipsychotics are also being used off-label to manage challenging behaviour among people with psychiatric diagnoses; however, we are unable to determine the indication for use in this population. It is also important to note that guidelines recommend the use of antipsychotics to manage challenging behaviour when evidence-based alternatives have been unsuccessful, and when the risk to the person or others is severe.$^{30}$ Using data available to us, we are unable to determine the alignment of antipsychotic use with these recommendations in this analysis. However, the high antipsychotic initiation rate, often with several other medications, and variability in use across comorbid profiles and practice settings suggest that considerable risk is being introduced to adults with IDD due to their use. Future research should investigate further the appropriateness of this prescribing and its association with harm. Third, our cohort was restricted to adults aged 18-64 years, and therefore, these findings may not be generalisable to youth or older adults. Fourth, we defined new antipsychotic use on the basis of no antipsychotic prescription in the prior year. Therefore, it is possible that some individuals in our cohort had more remote prior use of antipsychotics. Fifth, we are unable to study some variables that may be associated with antipsychotic initiation, including severity of intellectual disability, history of challenging behaviour and family and paid caregiver level of stress and attitudes towards medication use. Furthermore, we are unable to determine the degree of intellectual disability or to specifically investigate the subgroup of individuals within the cohort with an autism diagnosis. Future research should consider further exploration into antipsychotic initiation within subsets of the IDD population. Finally, we determined residence in a group home setting using data from 2009 and assumed that this did not change over the study period. Although residence in these settings is relatively stable in Ontario, it is possible that living arrangements for some members of our cohort could have changed over the study period. Several of these limitations could be addressed through the ongoing collection and linkage of provincial health and social services data which would provide researchers with accurate estimates of IDD prevalence, living arrangements, and eligibility for public drug programmes.

\section{CONCLUSION}

The findings of this study suggest that, not only is antipsychotic prescribing high among adults with IDD, but factors associated with the initiation of an antipsychotic differ according to the presence of a psychiatric diagnosis and certain comorbidities. Over one-quarter of antipsychotic initiation occurred in the absence of any psychiatric diagnosis, suggesting a noteworthy degree of off-label antipsychotic use to manage challenging behaviour in a population with a high comorbidity and medication burden. However, in contrast, physicians appear to be more cautious in their antipsychotic prescribing to patients with pre-existing metabolic conditions, suggesting an appreciation of the potential metabolic complications associated with their use. Given the long duration of antipsychotic use in these individuals, future research is needed to understand the appropriateness of antipsychotic initiation among adults with IDD, and the safety implications of long-term use of these products. Until such evidence is developed, opportunities must be found to improve clinician knowledge regarding guideline recommendations for use and monitoring of antipsychotics in this vulnerable population to ensure that these medications are not being used to broadly manage behavioural issues without adequate knowledge of their harmful effects.

Author affiliations

${ }^{1}$ Li Ka Shing Knowledge Institute, St. Michael's Hospital, Toronto, Ontario, Canada ${ }^{2}$ ICES, Toronto, Ontario, Canada 
${ }^{3}$ Leslie Dan Faculty of Pharmacy, University of Toronto, Toronto, Ontario, Canada ${ }^{4}$ Institute of Health Policy, Management, and Evaluation, University of Toronto, Toronto, Canada

${ }^{5}$ Department of Psychiatry, University of Toronto, Toronto, Ontario, Canada ${ }^{6}$ Department of Psychiatry, Women's College Hospital, Toronto, Ontario, Canada ${ }^{7}$ Department of Psychology, University of Ottawa, Ottawa, Ontario, Canada ${ }^{8}$ Azrieli Adult Neurodevelopmental Centre, Centre for Addiction and Mental Health, Toronto, Ontario, Canada

Contributors TG was responsible for conception and design of the study, data acquisition and interpretation and drafting of the manuscript. WK was responsible for design of the study, data acquisition, analysis and interpretation and critical revisions of the manuscript. MT, SV and VC were responsible for design of the study, data interpretation and critical revisions of the manuscript. YL was responsible for conception and design of the study, data acquisition and interpretation and drafting of the manuscript. All authors provided final approval of the manuscript.

Funding This study was funded by grants from the Province of Ontario. It was also supported by ICES, which is funded by an annual grant from the Ontario Ministry of Health and Long-Term Care (MOHLTC). We would like to thank IMS Brogan Inc for use of their Drug Information Database. Parts of this material are based on data and information compiled and provided by the Canadian Institute for Health Information (ClHI).

Disclaimer The opinions, results and conclusions reported in this paper are those of the authors and are independent from the funding source and those of $\mathrm{CIHI}$. No endorsement by ICES, the Ontario government, or $\mathrm{CIHI}$ is intended or should be inferred.

Competing interests None declared.

Patient consent for publication Not required.

Ethics approval This study was approved by the research ethics board of Sunnybrook Health Sciences Centre, Toronto and conducted at ICES in Toronto, Canada using deidentified data.

Provenance and peer review Not commissioned; externally peer reviewed.

Data sharing statement The data set from this study is held securely in coded form at ICES. While data sharing agreements prohibit ICES from making the data set publicly available, access may be granted to those who meet prespecified criteria for confidential access, available at www.ices.on.ca/DAS. The full data set creation plan and underlying analytic code are available from the authors upon request, understanding that the programmes may rely on coding templates or macros that are unique to ICES.

Open access This is an open access article distributed in accordance with the Creative Commons Attribution Non Commercial (CC BY-NC 4.0) license, which permits others to distribute, remix, adapt, build upon this work non-commercially, and license their derivative works on different terms, provided the original work is properly cited, appropriate credit is given, any changes made indicated, and the use is non-commercial. See: http://creativecommons.org/licenses/by-nc/4.0/.

\section{REFERENCES}

1. Holden B, Gitlesen JP. Psychotropic medication in adults with mental retardation: prevalence, and prescription practices. Res Dev Disabil 2004;25:509-21.

2. Hsu SW, Chiang PH, Chang YC, et al. Trends in the use of psychotropic drugs in people with intellectual disability in Taiwan: a nationwide outpatient service study, 1997-2007. Res Dev Disabil 2014;35:364-72.

3. Sheehan R, Hassiotis A. Reduction or discontinuation of antipsychotics for challenging behaviour in adults with intellectual disability: a systematic review. Lancet Psychiatry 2017;4:238-56.

4. Robertson J, Emerson E, Gregory N, et al. Receipt of psychotropic medication by people with intellectual disability in residential settings. J Intellect Disabil Res 2000;44(Pt 6):666-76.

5. Tsiouris JA, Kim SY, Brown WT, et al. Prevalence of psychotropic drug use in adults with intellectual disability: positive and negative findings from a large scale study. J Autism Dev Disord 2013;43:719-31.

6. de Kuijper G, Evenhuis H, Minderaa RB, et al. Effects of controlled discontinuation of long-term used antipsychotics for behavioural symptoms in individuals with intellectual disability. J Intellect Disabil Res 2014:58:71-83.
7. Sheehan R, Hassiotis A, Walters K, et al. Mental illness, challenging behaviour, and psychotropic drug prescribing in people with intellectual disability: UK population based cohort study. BMJ 2015;351:h4326.

8. Lunsky Y, Khuu W, Tadrous M, et al. Antipsychotic Use With and Without Comorbid Psychiatric Diagnosis Among Adults with Intellectual and Developmental Disabilities. Can J Psychiatry 2018;63.

9. Tyrer P, Oliver-Africano PC, Ahmed Z, et al. Risperidone, haloperidol, and placebo in the treatment of aggressive challenging behaviour in patients with intellectual disability: a randomised controlled trial. Lancet 2008;371:57-63.

10. Vigod SN, Lunsky Y, Cobigo V, et al. Morbidity and mortality of women and men with intellectual and developmental disabilities newly initiating antipsychotic drugs. BJPsych Open 2016;2:188-94.

11. Sheehan R, Horsfall L, Strydom A, et al. Movement side effects of antipsychotic drugs in adults with and without intellectual disability: UK population-based cohort study. BMJ Open 2017;7:e017406.

12. Glover G, Bernard S, Branford D, et al. Use of medication for challenging behaviour in people with intellectual disability. $\mathrm{Br} J$ Psychiatry 2014;205:6-7.

13. Lunsky Y, Klein-Geltink JE, Yates EA. Atlas on the primary care of adults with developmental disabilities in Ontario. Institute for Clinical Evaluative Sciences \& Centre for Addiction and Mental Health. Toronto: ON, 2013.

14. Hux JE, Ivis F, Flintoft V, et al. Diabetes in Ontario: determination of prevalence and incidence using a validated administrative data algorithm. Diabetes Care 2002;25:512-6.

15. Tu K, Campbell NR, Chen ZL, et al. Accuracy of administrative databases in identifying patients with hypertension. Open Med 2007:1:e18-26.

16. Austin PC, Daly PA, Tu JV, Jv T. A multicenter study of the coding accuracy of hospital discharge administrative data for patients admitted to cardiac care units in Ontario. Am Heart $J$ 2002;144:290-6.

17. Mast G, Fernandes K, Tadrous M, et al. Persistence of Antipsychotic Treatment in Elderly Dementia Patients: A Retrospective, PopulationBased Cohort Study. Drugs Real World Outcomes 2016;3:175-82.

18. Vigod SN, Gomes T, Wilton AS, et al. Antipsychotic drug use in pregnancy: high dimensional, propensity matched, population based cohort study. BMJ 2015;350:h2298.

19. Rochon PA, Normand SL, Gomes T, et al. Antipsychotic therapy and short-term serious events in older adults with dementia. Arch Intern Med 2008;168:1090-6.

20. Steele LS, Glazier RH, Lin E, et al. Using administrative data to measure ambulatory mental health service provision in primary care. Med Care 2004;42:960-5.

21. Kisely S, Lin E, Gilbert C, et al. Use of administrative data for the surveillance of mood and anxiety disorders. Aust $N$ Z J Psychiatry 2009;43:1118-25.

22. Quan H, Sundararajan V, Halfon P, et al. Coding algorithms for defining comorbidities in ICD-9-CM and ICD-10 administrative data. Med Care 2005;43:1130-9.

23. Mamdani M, Sykora K, Li P, et al. Reader's guide to critical appraisal of cohort studies: 2. Assessing potential for confounding. BMJ 2005;330:960-2.

24. National Core Indicators. What does $\mathrm{NCI}$ tell us about adults with intellectual and developmental disabilities who are taking prescribed medications for anxiety, behavior challenges, mood disorders or psychotic disorders? 2012.

25. Lunsky Y, Elserafi J. Antipsychotic medication prescription patterns in adults with developmental disabilities who have experienced psychiatric crisis. Res Dev Disabil 2012;33:32-8.

26. Paton C, Bhatti S, Purandare K, et al. Quality of prescribing of antipsychotic medication for people with intellectual disability under the care of UK mental health services: a cross-sectional audit of clinical practice. BMJ Open 2016;6:e013116.

27. O'Dwyer M, Peklar J, Mulryan N, et al. Prevalence, patterns and factors associated with psychotropic use in older adults with intellectual disabilities in Ireland. J Intellect Disabil Res 2017;61:969-83.

28. Frighi V, Stephenson MT, Morovat A, et al. Safety of antipsychotics in people with intellectual disability. Br J Psychiatry 2011;199:289-95.

29. Teeluckdharry S, Sharma S, O'Rourke E, et al. Monitoring metabolic side effects of atypical antipsychotics in people with an intellectual disability. J Intellect Disabil 2013;17:223-35.

30. National Institute for Health and Care Excellence. Challenging behaviour and learning disabilities: prevention and interventions for people with learning disabilities whose behaviour challenges. 2015 https://www.nice.org.uk/guidance/ng11 (accessed 24 May 2018). 